\title{
Routing Optimization in Wireless Mesh Networks under Uncertain Traffic Demands
}

\author{
Yang Song, Chi Zhang and Yuguang Fang \\ Department of Electrical and Computer Engineering \\ University of Florida \\ Gainesville, Florida 32611 \\ Email: \{yangsong@, zhangchi@ and fang@ece.\}ufl.edu
}

\begin{abstract}
In this paper, we investigate the routing optimization problem in wireless mesh networks. While existing works usually assume static and known traffic demand, we emphasize that the actual traffic is time-varying and difficult to measure. In light of this, we alternatively pursue a stochastic optimization framework where the expected network utility is maximized. For multi-path routing scenario, we propose a stochastic programming approach which requires no priori knowledge on the probabilistic distribution of the traffic. For the single-path routing counterpart, we develop a learningbased algorithm which provably converges to the global optimum solution asymptotically.
\end{abstract}

\section{Categories and Subject Descriptors}

C.2.1 [Network Architecture and Design]: Wireless Communications

\section{General Terms}

Algorithms, Design, Performance

\section{Keywords}

Wireless Mesh Networks, Routing, Learning Algorithms

\section{INTRODUCTION}

Wireless mesh networks provide last mile broadband Internet access with low cost yet high bandwidth. The mesh routers communicate with each other via wireless links. Meanwhile, several edge mesh routers provide wireless access to the client users where all the traffic is directed to the gateway node. Due to the multihop nature, the routing in wireless mesh networks is an important and interesting topic and has attracted significant attention from the community [1].

*Fang is also a Changjiang Scholar Chair Professor with National Key Laboratory of Integrated Services Networks, Xidian University, Xi'an, China.
However, the existing routing schemes in wireless mesh networks usually assume that the traffic demands aggregated at edge routers are static and known. In practice, apparently, the instantaneous traffic demand may fluctuate dramatically due to the client users' behavior and the mobility of users. Therefore, the uncertainty of the traffic demands at edge routers is not negligible and needs to be considered meticulously in wireless mesh networks.

Traffic estimation is proposed as a feasible solution to harness the traffic uncertainty. Several prediction models are proposed in the literature $[18,20,30]$. However, we emphasize that the acquisition of accurate traffic information is by no means trivial and usually computationally expensive. Even worse yet, the estimation techniques yield poor performance in a highly dynamic environment. Therefore, routing design in wireless mesh networks with uncertain traffic demand is remarkably challenging.

To circumvent the burden of route recalculation, oblivious routing schemes are suggested $[2,16,17,24,25]$. A traffic-oblivious routing protocol requires limited traffic information and achieves worst-case performance guarantee. In other words, by sticking to a fixed routing strategy, the worst-case performance is optimized. Therefore, the instability and prohibitive overhead of rerouting are avoided and the performance is acceptable if the traffic pattern varies within a certain range. However, one noticeable drawback of oblivious routing schemes is the computational complexity [25]. In addition, the optimization on the worst-case performance is usually over-conservative. Also, limited knowledge is attained when the traffic pattern varies drastically and exceeds the tolerance bound. In light of these concerns, we propose a stochastic optimum routing strategy in wireless mesh networks where the expected network utility is optimized, rather than the worst-case performance. Our schemes differ from previous work in several aspects. First, our algorithm does not require a priori knowledge of the traffic distribution. Therefore, the computational overhead of traffic estimation can be avoided. Secondly, our scheme does not require that the traffic pattern varies within a certain range. Thirdly, our proposed algorithms are amenable to decentralized implementations.

The remainder of this paper is organized as follows. Section 2 briefly outlines the related work. The system model used in this paper is introduced in Section 3. The routing optimization problems with multi-path and single-path routing constraints are investigated in Section 4 and Section 5, respectively. A numerical example is provided in Section 6 and Section 7 concludes this paper.

\section{RELATED WORK}

Routing plays a critical role in wireless mesh networks. In the literature, the routing problem is usually formulated as an optimization problem where heuristic or decomposition-based solutions are proposed. The existing works usually assume that the traffic input
QShine 2008, July 28-31, 2008, Hong Kong, Hong Kong. Copyright 2008 ICST ISBN 978-963-9799-26-4

DOI 10.4108/ICST.QSHINE2008.3841 
from each edge router is static and known. Meanwhile, the empirical studies based on real traffic traces reveal that the instantaneous traffic fluctuates from time to time and is difficult to predict. To address the uncertainty of traffic demand in mesh networks, two lines of research effort have been discussed. The first category, which is in analogy of robust optimization techniques, achieves optimal worst-case performance. Oblivious routing schemes fall into this category. The second category, which is in analogy of the general stochastic optimization techniques, alternatively pursues the expected utility maximization [6], [29] [13-15]. Our work falls into this category. In [14], the power scheduling problem with time-varying channel is investigated. In [29], the impact of noisy feedback is analyzed, where the traffic demand is assumed to be sufficiently large and a rate control algorithm, based on the dual decomposition approach, is implemented to adjust the ingress traffic. Nevertheless, in our work, the traffic demand is dynamic and may even appear zero sometime. Another related work is [6] where the throughput maximization routing is considered. The traffic dynamic in wireless mesh networks is well addressed. However, the authors assume the traffic statistics are known, i.e., the probability distribution of random traffic demand is assumed to be a priori. In our scheme, such information is not required, as will be clarified in following sections.

\section{MODEL}

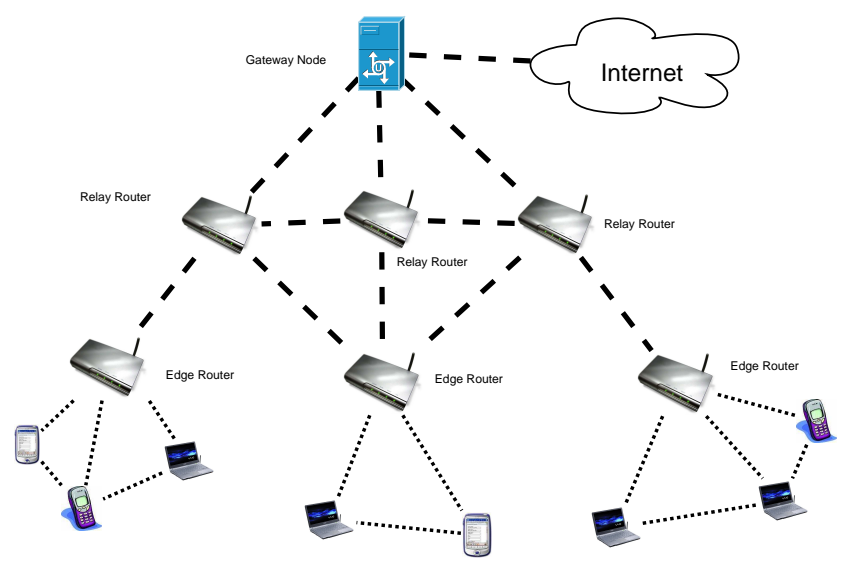

Figure 1: Wireless Mesh Network Topology.

We consider a wireless mesh network depicted in Figure 1. The mesh network consists of several edge routers, intermediate relay routers and a gateway node $\mathcal{W}$ which provides the connection to the Internet. Each edge router is associated with a number of client users. Denote the set of edge routers as $\mathcal{M}$ and the aggregated traffic demand at the $m$-th edge router as $z_{m}$. Naturally, $z_{m}$ is a random variable which is determined by the behaviors of client users. We assume that the instantaneous traffic demand at an edge router forms a stationary stochastic process.

There are two types of routing protocols in the literature, i.e., arc-routing and path-routing. In arc-routing protocols, the decision variable is the amount of traffic allocated on each link of the network, whereas the path-routing protocols adjust the fraction of traffic allocated on each distinct path. We follow the latter approach due to its practical merit [16]. For example, we can utilize the path diversity provided by OSPF/IS-IS and MPLS techniques. More specifically, OSPF divides the traffic evenly among all paths with the same cost whereas MPLS enables more flexible manipulation on the flows on all available paths. In our scenario, one edge router, say $m$, has $\mathcal{P}_{m}$ acyclic paths to the gateway node $\mathcal{W}$. The instantaneous traffic $z_{m}$ is distributed among all $\mathcal{P}_{m}$ paths and each path possesses a fraction, denoted by $r_{m}^{k}$ where $^{1} m=1, \cdots, \mathcal{M}$ and $k=1, \cdots, \mathcal{P}_{m}$. We have

$$
\sum_{k=1}^{\mathcal{P}_{m}} r_{m}^{k} \leq 1, \forall m \in \mathcal{M} .
$$

Define $\mathbf{r}_{m}$ as the fraction vector and $\mathbf{r}=\left[\mathbf{r}_{1}, \cdots, \mathbf{r}_{\mathcal{M}}\right]$, which is the decision variable in our optimization framework throughout the paper. We define

$$
\delta_{m}=\sum_{k=1}^{\mathcal{P}_{m}} r_{m}^{k}, \forall m \in \mathcal{M}
$$

for notation succinctness.

We denote the set of wireless links in the mesh network as $\mathcal{E}$. We assume in this paper that a scheduling scheme is available for the medium access where each link possesses a time share of the channel access. For each link $e \in \mathcal{E}$, there is a link cost, denoted by $l_{e}$, which is a function of the instantaneous aggregated flow and the achievable data rate of the link, denoted by $f_{e}$ and $c_{e}$, respectively. Note that $c_{e}$ is given by

$$
c_{e}=\tilde{c}_{e} \times \gamma_{e}
$$

where $\tilde{c}_{e}$ denotes the nominal capacity of the link and $\gamma_{e}$ is the fraction of time that link $e$ is active following the scheduling. Without loss of generality, we assume the power of each link is fixed.

Each edge router has a concave utility function, denoted by $U\left(x_{m}\right)$, which reflects the degree of satisfaction by transmitting a flow of $x_{m}=z_{m} \times \delta_{m}$. In light of the link costs, the surplus of the $m$-th edge router, a.k.a., net reward, is given by

$$
O_{m}=U_{m}\left(z_{m} \times \delta_{m}\right)-\sum_{k=1}^{\mathcal{P}_{m}} z_{m} r_{m}^{k} \sum_{e \in P_{m}^{k}} l_{e}\left(f_{e}\right)
$$

where $e \in P_{m}^{k}$ represents all links along the $k$-th path of $\mathcal{P}_{m}$. We assume that the link cost function $l_{e}\left(f_{e}\right)$ is a non-decreasing and differentiable function of $f_{e}$. Two such well-known examples are

$$
l_{e}\left(f_{e}\right)=\frac{1}{c_{e}-f_{e}}
$$

which represents the average waiting time on link $e$, and

$$
l_{e}\left(f_{e}\right)=\frac{f_{e}}{\left(c_{e}-f_{e}\right) c_{e}}
$$

which reflects the average queueing time on link $e$, following a model of $M / M / 1$ [12]. Note that the cost is considered as $+\infty$ if $f_{e} \geq c_{e}$.

Define a topology matrix $H_{m}$ for the $m$-th edge router where the element $H_{e, k}^{m}=1$ represents that link $e$ is on the $k$-th path of $\mathcal{P}_{m}$ and zero otherwise. Therefore, we have

$$
f_{e}=\sum_{m=1}^{\mathcal{M}} \sum_{k=1}^{\mathcal{P}_{m}} H_{e, k}^{m} z_{m} r_{m}^{k}
$$

Note that in wireless mesh networks, the edge routers and relay routers are usually static. Therefore, the topology matrix, i.e., $H_{m}$, is a fixed binary matrix and can be acquired easily. However, the

\footnotetext{
${ }^{1}$ In this paper, we slightly abuse the notation by using the same symbol for a set and its cardinality for notation succinctness.
} 
values of $f_{e}, z_{m}$ and $l_{e}$ are random due the uncertain traffic demand, which makes the routing problem more challenging. In Section 4 , we formulate the multi-path routing optimization problem of wireless mesh networks in a stochastic programming framework where a distributed algorithmic solution is derived. The single-path routing scenarios are investigated in Section 5, where a learningbased algorithm is introduced to achieve the global optimum solution asymptotically.

\section{MULTI-PATH ROUTING SCENARIO}

In this section, we consider the cases where multi-path routing is allowed for fault tolerance and load balancing purpose, i.e., the aggregated flow of each edge router is distributed among several available paths.

In the network, each edge router has a surplus given by (4). From the network's perspective, our objective is to maximize the overall surplus of the network, i.e., $\sum_{m=1}^{\mathcal{M}} O_{m}$. However, due to the randomness induced by the traffic demand uncertainty, the overall surplus itself is a random variable. Therefore, we alternatively pursue a stochastic optimum solution of $\mathbf{r}^{*}$, which maximizes the expected overall surplus of the network. Mathematically, the routing problem in the wireless mesh network is formulated as

$$
\begin{aligned}
& \underset{\mathbf{r}}{\max _{m}} \mathbb{E}\left(\sum_{m=1}^{\mathcal{M}} O_{m}\right) \\
& \quad \mathbb{E}\left(f_{e}\right) \leq c_{e} \forall e \in \mathcal{E} \\
& \quad \sum_{k=1}^{\mathcal{P}_{m}} r_{m}^{k} \leq 1 \forall m \in \mathcal{M} \\
& \quad f_{e}=\sum_{m=1}^{\mathcal{M}} \sum_{k=1}^{\mathcal{P}_{m}} H_{e, k}^{m} z_{m} r_{m}^{k} \forall e \in \mathcal{E} \\
& \quad O_{m}=U_{m}\left(z_{m} \times \delta_{m}\right)-\sum_{k=1}^{\mathcal{P}_{m}} z_{m} r_{m}^{k} \sum_{e \in P_{m}^{k}} l_{e}\left(f_{e}\right) \forall m \in \mathcal{M}
\end{aligned}
$$

where $\mathbb{E}($.$) is the expectation operator. We assume that the random$ traffic demands of all edge routers can be discretized into arbitrarily many yet finite states, where each state is represented by $s \in \mathcal{S}$. The time is slotted and within one slot, the traffic demands on all edge routers correspond to one of the states and remain unchanged within the current slot. Note that $O_{m}, f_{e}, l_{e}$ and $z_{m}$ are all statedependent variables and hence we will add a superscript $s$ in the following formulations. Denote the stationary probability distribution of state $s$ as $\pi_{s}$. We can rewrite the routing optimization problem as

$$
\begin{aligned}
& \max _{\mathbf{r}} \sum_{s \in S} \pi_{s}\left(\sum_{m=1}^{\mathcal{M}} O_{m}^{s}\right) \\
& \text { s.t. } \\
& \sum_{s \in S} \pi_{s}\left(f_{e}^{s}\right) \leq c_{e} \forall e \in \mathcal{E} \\
& \sum_{k=1}^{\mathcal{P}_{m}} r_{m}^{k} \leq 1 \forall m \in \mathcal{M} \\
& f_{e}^{s}=\sum_{m=1}^{\mathcal{M}} \sum_{k=1}^{\mathcal{P}_{m}} H_{e, k}^{m} z_{m}^{s} r_{m}^{k} \forall e \in \mathcal{E} \\
& O_{m}^{s}=U_{m}\left(z_{m}^{s} \times \delta_{m}\right)-\sum_{k=1}^{\mathcal{P}_{m}} z_{m}^{s} r_{m}^{k} \sum_{e \in P_{m}^{k}} l_{e}^{s}\left(f_{e}^{s}\right) \forall m \in \mathcal{M}
\end{aligned}
$$

We can verify that if the stationary probability distribution $\pi_{s}$ is known as a priori, the optimization problem is a deterministic convex optimization problem and the Slater's condition is satisfied. However, the actual value of $\pi_{s}$ is either difficult to measure in practice, or needs significant computational overhead to estimate. Next, we propose a distributed solution which converges to the global optimum solution yet requires no information about the underlying probabilistic distribution.

We first obtain the Lagrangian as

$$
\begin{aligned}
& L(\mathbf{r}, \lambda, \mu) \\
= & \sum_{s \in S} \pi_{s}\left\{\sum_{m \in \mathcal{M}} U_{m}\left(z_{m}^{s} \times \delta_{m}\right)-\sum_{m \in \mathcal{M}} \sum_{k \in \mathcal{P}_{m}} z_{m}^{s} r_{m}^{k}\left(\sum_{e \in P_{m}^{k}} l_{e}^{s}\left(f_{e}^{s}\right)\right)\right\} \\
+ & \sum_{m \in \mathcal{M}} \lambda_{m}\left(1-\sum_{k \in \mathcal{P}_{m}} r_{m}^{k}\right)+\sum_{e \in \mathcal{E}} \mu_{e}\left(c_{e}-\sum_{s \in S} \pi_{s} f_{e}^{s}\right) \\
= & \sum_{s \in S} \pi_{s}\left\{\sum_{m \in \mathcal{M}}\left(U_{m}\left(z_{m}^{s} \times \delta_{m}\right)+\lambda_{m}\right)+\sum_{e \in \mathcal{E}} \mu_{e} c_{e}\right. \\
& -\sum_{m \in \mathcal{M}} \sum_{k \in \mathcal{P}_{m}}\left(z_{m}^{s} r_{m}^{k}\left(\sum_{e \in P_{m}^{k}}\left(l_{e}^{s}\left(f_{e}^{s}\right)+\mu_{e}\right)+\lambda_{m} r_{m}^{k}\right)\right\}
\end{aligned}
$$

Note that $\mu$ is the link congestion price similar as $[4,5,10]$. The dual variables of $\lambda$ ensure that the summation of fraction variables is less or equal to unity. Define

$$
\begin{aligned}
Q^{s}= & \sup _{\mathbf{r}}\left\{\sum_{m \in \mathcal{M}}\left(U_{m}\left(z_{m}^{s} \times \delta_{m}\right)+\lambda_{m}\right)+\sum_{e \in \mathcal{E}} \mu_{e} c_{e}\right. \\
& -\sum_{m \in \mathcal{M}} \sum_{k \in \mathcal{P}_{m}}\left(z_{m}^{s} r_{m}^{k}\left(\sum_{e \in P_{m}^{k}}\left(l_{e}^{s}\left(f_{e}^{s}\right)+\mu_{e}\right)+\lambda_{m} r_{m}^{k}\right)\right\}
\end{aligned}
$$

Therefore, the dual function is given by

$$
g(\lambda, \mu)=\sum_{s \in S} \pi_{s} Q^{s} \text { where } \lambda \geq 0, \mu \geq 0 .
$$

To achieve the minimum value of (18), or equivalently, to obtain the stochastic optimum value of $\mathbf{r}^{*}$, we utilize the stochastic primaldual approach [5]. The dual variables, i.e., $\lambda$ and $\mu$ are updated as

$$
\begin{aligned}
\lambda_{m}(n+1) & =\left[\lambda_{m}(n)-\alpha_{m}(n) \zeta_{m}(n)\right]^{+} \forall m \in \mathcal{M} \\
\mu_{e}(n+1) & =\left[\mu_{e}(n)-\alpha_{e}(n) \xi_{e}(n)\right]^{+} \forall e \in \mathcal{E}
\end{aligned}
$$


where $[x]^{+}$denotes $\max (x, 0)$. Similarly, the primal variable, i.e., $r_{m}^{k}$, is updated as

$$
r_{m}^{k}(n+1)=\left[r_{m}^{k}(n)+\alpha_{m, k}(n) \eta_{m, k}(n)\right]_{0}^{1}
$$

where $[x]_{a}^{b}$ denotes $\max (\min (b, x), a)$ and we use symbol $\alpha(n)$ to represent the corresponding stepsizes, i.e., $\alpha_{m}(n), \alpha_{e}(n)$ and $\alpha_{m, k}(n)$, generally. Note that the instantaneous link flow is considered as fixed when the fraction variable $r_{m}^{k}$ is updated.

The stochastic subgradients, i.e., $\zeta_{m}(n), \xi_{e}(n)$ and $\eta_{m, k}(n)$, can be attained by the Danskin's theorem [3], following a similar approach as in $[13,14]$

$$
\begin{aligned}
& \zeta_{m}(n)=1-\sum_{k \in \mathcal{P}_{m}} r_{m}^{k} \forall m \in \mathcal{M} \\
& \xi_{e}(n)=c_{e}-\sum_{m \in \mathcal{M}} \sum_{k \in \mathcal{P}_{m}} z_{m}^{s} r_{m}^{k} H_{e, k}^{m} \forall e \in \mathcal{E} \\
& \eta_{m, k}(n)=-\left[\lambda_{m}+z_{m}^{s}\left(\sum_{e \in P_{m}^{k}}\left(l_{e}^{s}+\mu_{e}\right)\right)\right] \forall m \in \mathcal{M} .
\end{aligned}
$$

The distributed implementation of the algorithm is summarized as follows.

\section{Algorithm:}

\section{Repeat:}

- Each link measures

$$
\xi_{e}(n)=c_{e}-\sum_{m \in \mathcal{M}} \sum_{k \in \mathcal{P}_{m}} z_{m}^{s} r_{m}^{k} H_{e, k}^{m}
$$

- Each link updates the link congestion price as

$$
\mu_{e}(n+1)=\left[\mu_{e}(n)-\alpha_{e}(n) \xi_{e}(n)\right]^{+} .
$$

- Each edge router measures

$$
\zeta_{m}(n)=1-\sum_{k \in \mathcal{P}_{m}} r_{m}^{k}
$$

and

$$
\eta_{m, k}(n)=-\left[\lambda_{m}+z_{m}^{s}\left(\sum_{e \in P_{m}^{k}}\left(l_{e}^{s}+\mu_{e}\right)\right)\right] .
$$

- Each edge router updates the fraction variable as

$$
r_{m}^{k}(n+1)=\left[r_{m}^{k}(n)+\alpha_{m, k}(n) \eta_{m, k}(n)\right]_{0}^{1} .
$$

- Each edge router updates the dual variable $\lambda$ as

$$
\lambda_{m}(n+1)=\left[\lambda_{m}(n)-\alpha_{m}(n) \zeta_{m}(n)\right]^{+} .
$$

Until:

- The difference between successive iterations, i.e.,

$$
\epsilon=\left|r_{m}^{k}(n+1)-r_{m}^{k}(n)\right|
$$

is within a predefined convergence threshold $\epsilon^{\prime}$.

\section{End}

Note that the information needed in the algorithm is either locally attainable or acquirable by the feedback along the paths. Therefore, the algorithm is amenable to distributed implementation. The convergence of the algorithm follows the results of [29] and thus we provide the following theorem without proof.
THEOREM 1. The algorithm converges to the global optimum with probability one, provided that the following constraints are satisfied [9,29]: $\alpha(n)>0, \sum_{n=0}^{\infty} \alpha(n)=\infty, \lim _{n \rightarrow \infty} \alpha(n)=0$ and $\sum_{n=0}^{\infty}(\alpha(n))^{2}<\infty, \forall m \in \mathcal{M}$ and $e \in \mathcal{E}$, where $\alpha$ generally represents all stepsize parameters in (19), (20) and (21).

\section{SINGLE-PATH ROUTING SCENARIO}

In this section, we consider a wireless mesh network where singlepath routing strategy is adopted. Therefore, the fraction variable $r_{m}^{k}$ is a binary number in this scenario. More specifically, $r_{m}^{k}=1 \mathrm{de}-$ notes that the $m$-th edge router selects the $k$-th path to deliver traffic and the fraction variables on other paths are zeros. The integral property of the fraction variables complicates the routing optimization problem. We first express the routing optimization problem, with single path routing constraint, as

$$
\begin{aligned}
& \max _{\mathbf{r}} \sum_{s \in S} \pi_{s}\left(\sum_{m=1}^{\mathcal{M}} O_{m}^{s}\right) \\
& \quad \sum_{s \in S} \pi_{s}\left(f_{e}^{s}\right) \leq c_{e} \forall e \in \mathcal{E} \\
& \quad \sum_{k=1}^{\mathcal{P}_{m}} r_{m}^{k} \leq 1 \forall m \in \mathcal{M} \\
& \quad f_{e}^{s}=\sum_{m=1}^{\mathcal{M}} \sum_{k=1}^{\mathcal{P}_{m}} H_{e, k}^{m} z_{m}^{s} r_{m}^{k} \forall e \in \mathcal{E} \\
& \quad O_{m}^{s}=U_{m}\left(z_{m}^{s} \times \delta_{m}\right)-\sum_{k=1}^{\mathcal{P}_{m}} z_{m}^{s} r_{m}^{k} \sum_{e \in P_{m}^{k}} l_{e}^{s}\left(f_{e}^{s}\right) \forall m \in \mathcal{M} \\
& \\
& \quad r_{m}^{k} \in\{0,1\}
\end{aligned}
$$

Apparently, this is a stochastic integer programming which is difficult and computationally demanding to solve. For a survey on the algorithmic solutions of stochastic integer programming problems, refer to [23]. Distinguishing from previous work, we next propose a learning-based algorithm, which asymptotically converges to the global optimum of the aforementioned stochastic integer programming problem. First, we briefly overview the learning automata technique, based on which our algorithm is proposed.

\subsection{Learning Automata}

Learning automata techniques have been broadly investigated in the networking community [26] [8] [7] [11] [27] [19]. As one of the stochastic learning schemes, learning automata was first introduced in the control community for stochastic systems. As depicted in Figure 2, the basic single user scenario where learning automata techniques can be applied consists of a random environment, a set of finite actions and a rational decision maker. At a time instance, the decision maker selects one of the actions according to the selection probability vector $\mathbf{p}$. The random environment responds with a stochastic output based on which the learning algorithm updates the selection probability vector and the iteration continues. In a stationary random environment, the standard learning automata algorithms asymptotically converge to an action which is stochastically optimal in the sense that the expected objective is maximized [22].

In our scenario, each edge router is an independent decision maker. The action space corresponds to the available paths of each edge router. Meanwhile, the random environment is the uncertain 


\section{Random Environment}

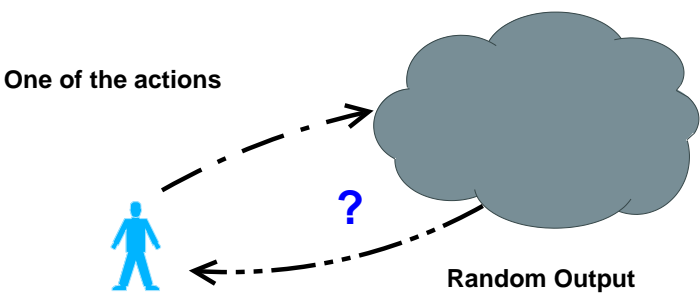

Decision Maker

Figure 2: Structure of A Single User Learning Automata.

traffic demands at all edge routers jointly. To incorporate a multiagent learning scenario, we form all $\mathcal{M}$ edge routers as a learning team, which targets to the stochastic optimum solution collectively. In addition, we utilize the gateway node $\mathcal{W}$ of the wireless mesh network as a teacher. After each decision maker selects an action, the gateway node multicasts a feedback signal, denoted by $\beta$, based on which each edge router updates the selection probability vector and the algorithm iterates until convergence.

\subsection{Learning-based Algorithm}

Each edge router, say $m$, maintains a selection probability vector $\mathbf{p}_{m}$ and an inner-state vector $\mathbf{u}_{m}$. We use the notation $(m, k)$ to indicate the $k$-th path of the $m$-th edge router, i.e., the $k$-th action from the action space of the decision maker $m$.

At the initialization phase, the value of $\mathbf{u}_{m}$ is randomly generated and $\mathbf{p}_{m}=\left[1 / \mathcal{P}_{m}, \cdots, 1 / \mathcal{P}_{m}\right]$. At time instance $n$, the algorithm executed at the $m$-th edge router is described as follows.

\section{Algorithm:}

\section{Repeat:}

- Selects a routing path from $\mathcal{P}_{m}$, say $j$, according to the selection probability vector $\mathbf{p}_{m}(n)$ and starts the transmission.

- After receiving the feedback signal from the gateway node $\mathcal{W}$, denoted by $\beta(n)$, the inner state vector $\mathbf{u}_{m}$ is updated as

$$
\begin{aligned}
u_{m, k}(n+1)= & {\left[u_{m, k}(n)+\theta(n) \beta(n)\left(1-\frac{e^{u_{m, k}}}{\sum_{k \in \mathcal{P}_{m}} e^{u_{m, k}}}\right)\right.} \\
& \left.+\sqrt{\theta(n)} \omega_{m, k}(n)\right]_{0}^{\mathcal{L}}, \text { for } k=j \\
u_{m, k}(n+1)= & {\left[u_{m, k}(n)+\sqrt{\theta(n)} \omega_{m, k}(n)\right]_{0}^{\mathcal{L}} \text { for } k \neq j }
\end{aligned}
$$

Recall that $j$ denotes the selected action, i.e., the chosen path.

- The selection probability vector $\mathbf{p}_{m}$ is then updated, following

$$
p_{m, k}=\frac{e^{u_{m, k}}}{\sum_{k \in \mathcal{P}_{m}} e^{u_{m, k}}} \forall k \in \mathcal{P}_{m} .
$$

Until:

$\max \left(\mathbf{p}_{m}(n)\right)>B$ where $B$ is a predefined convergence threshold.
In the algorithm, $\theta(n)$ is the learning parameter of the algorithm satisfying $0<\theta(n)<1$. $\mathcal{L}$ is a positive number which keeps the inner state value bounded. The sequence of $\omega_{m, k}(n)$ is a set of random variables with zero mean and a variance of $\sigma^{2}(n)$. The global feedback signal $\beta(n)$ is calculated by the gateway node $\mathcal{W}$ as

$$
\beta(n)=\frac{\sum_{m=1}^{\mathcal{M}} O_{m}^{s}}{\mathcal{J}}
$$

where $\mathcal{J}$ is a sufficiently large number to normalize the output. In other words, the value of $\beta(n)$ is deliberately tuned within $[0,1]$. We emphasize that the introduced noise parameter $\omega$ restrains the algorithm from being trapped in an inefficient equilibrium. Note that the value of $\beta(n)$ can be informed by efficient multicast algorithms, e.g., [28], initiated by the gateway node $\mathcal{W}$. The team learning is then executed in a decentralized fashion. The steady state behavior of the learning-based algorithm is given in the following theorem.

THEOREM 2. The proposed learning-based algorithm converges to the global optimum solution of the single-path routing optimization problem with probability one, if the following conditions are satisfied [21]: (1) $\lim _{n \rightarrow \infty} \theta(n)=0$ and (2) $\lim _{n \rightarrow \infty} \sigma(n)=0$.

The proof of the theorem benefits from the stochastic differential equation (SDE) approach which follows similar lines as [21] and thus omitted due to the page limit. In the next section, we will demonstrate the efficacy of our algorithmic approaches via a numerical example, for both multi-path and single-path scenarios.

\section{NUMERICAL EXAMPLE}

We consider an illustrative wireless mesh network shown in Figure 3. Among all available paths, we assign a set of paths for each edge router and the corresponding links ${ }^{2}$ are shown in Figure 3.

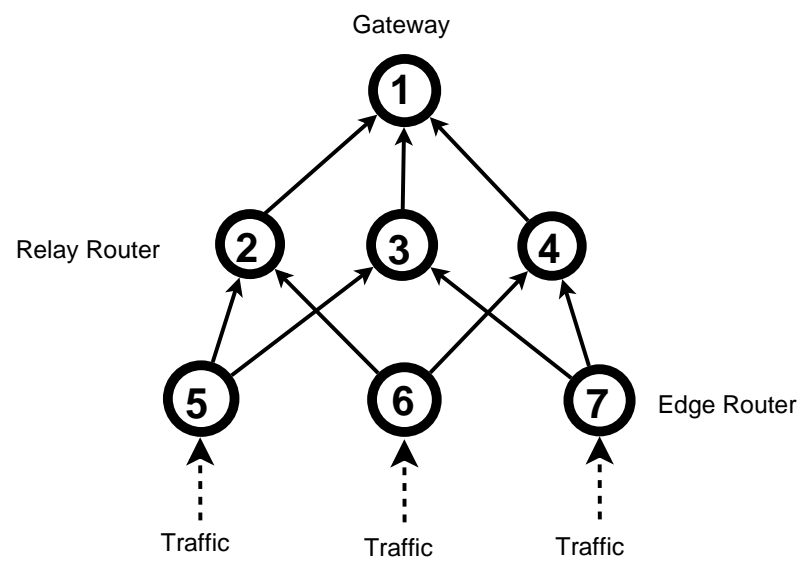

Figure 3: Illustration of Available Paths.

The gateway node is indicated as node 1 . Three edge routers are marked as node 5,6 and 7 , respectively, which consistently deliver the aggregated traffic to the gateway node. The available paths for each edge router are provided in Table I.

Note that each edge router has two acyclic paths to reach the destination. The link cost function follows (5). The traffic is randomly generated at each edge router with Gaussian distribution. The mean

\footnotetext{
${ }^{2}$ Note that the actual network topology can be much larger.
} 
Table 1: Available paths for each edge router.

\begin{tabular}{||c|c|c||}
\hline Edge router 5 & $P_{5}^{1}:$ & $\{5 \rightarrow 2 \rightarrow 1\}$ \\
\cline { 2 - 3 } & $P_{5}^{2}:$ & $\{5 \rightarrow 3 \rightarrow 1\}$ \\
\hline \multirow{2}{*}{ Edge router 6} & $P_{6}^{1}:$ & $\{6 \rightarrow 2 \rightarrow 1\}$ \\
\cline { 2 - 3 } & $P_{6}^{2}:$ & $\{6 \rightarrow 4 \rightarrow 1\}$ \\
\hline \multirow{2}{*}{ Edge router 7} & $P_{7}^{1}:$ & $\{7 \rightarrow 3 \rightarrow 1\}$ \\
\cline { 2 - 3 } & $P_{7}^{2}:$ & $\{7 \rightarrow 4 \rightarrow 1\}$ \\
\hline
\end{tabular}

is 5 and the variance is 1 . In addition, we limit the traffic to be in the range of $[0,10]$. We emphasize that these settings do not involve any loss of generality. By setting the traffic of each router with identical statistical characteristics, the stochastic optimum solution coincides with the load-balancing solution. The computational difficulty of calculating the global optimum of the stochastic integer programming problem can be avoided. Therefore, we can easily verify the efficacy of our proposed algorithms. In addition, the actual achievable data rate is a function of the transmission power and the scheduling algorithm. Without loss of generality, we assume that the achievable data rate of each link is 10 . We utilize the same stepsize for the stochastic gradient approach in (19), (20) and (21), which is inversely proportional to the number of iterations.

We first investigate the multi-path routing scenario where each edge router can divide the incoming traffic on two available paths. Restated, it is straightforward to verify that, given the current settings of link achievable rates and statistical characteristics, the stochastic optimum solution coincides with the load-balancing solution. In other words, the solution of $r_{5}^{1}=r_{5}^{2}=r_{6}^{1}=r_{6}^{2}=r_{7}^{1}=r_{7}^{2}=\frac{1}{2}$ maximizes the expected overall network surplus and thus solves the routing optimization problem. This observation is verified in Figure 4.

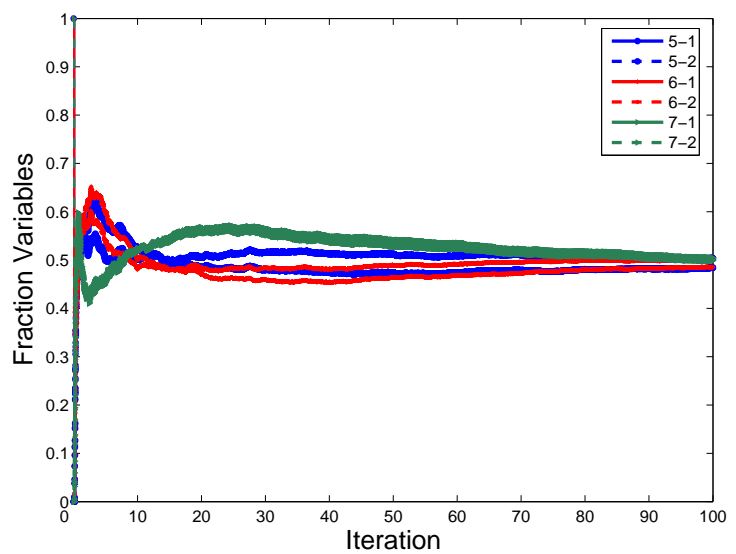

Figure 4: Convergence of Fraction Variables.

Figure 4 depicts the evolution of 6 fraction variables of edge routers where pair $m-k$ denotes $r_{m}^{k}$. At initialization, we manually set the fraction variables as $r_{5}^{1}=1, r_{5}^{2}=0, r_{6}^{1}=1, r_{6}^{2}=0$ and $r_{7}^{1}=0, r_{7}^{2}=1$ which is remarkably biased. Then, the distributed algorithm derived in Section 4 is executed. As pictorially shown by Figure 4, all 6 fraction variables evolve with the iterations and converge to the stochastic optimum solution gradually.

Next, we investigate the single-path routing scenario. The network settings are the same as in multi-path scenarios except that each edge router can only select one of the available paths to trans- mit. The learning parameter is set to $\theta(n)=1 / n$ where $n$ is the index of the current iteration. The noise parameters are zero mean Gaussian random variables with diminishing variances, e.g., $\sigma(n)=1 / n$. The bounding parameter $\mathcal{L}$ is 100 and $\mathcal{J}$ is 100 . The convergence threshold $B$ is 0.9999 . The learning-based algorithm is executed until each edge router sticks to one of the available routing paths. The convergence behavior of the learning-based algorithm is demonstrated in Figure 5.

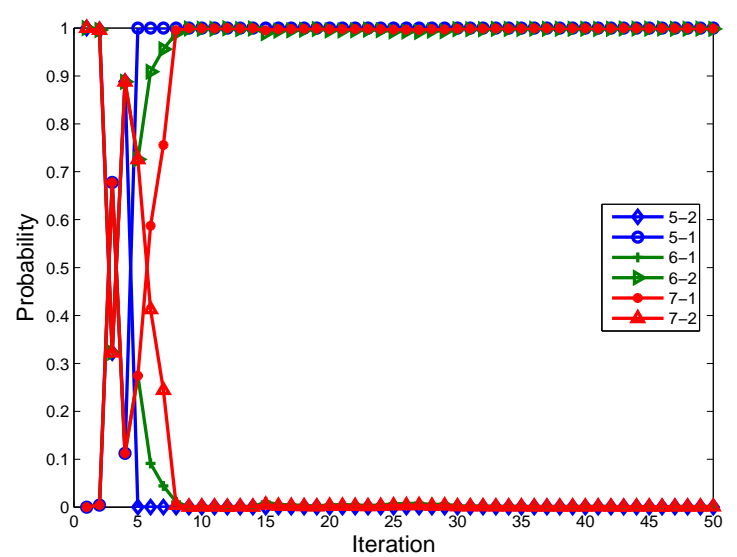

Figure 5: Convergence of Selection Probability Vectors.

In Figure 5, we denote pair $m-k$ as the probability that the $m$-th edge router picks the $k$-th path for next iteration. For example, $5-1$ represents the probability that edge router 5 selects the first path, i.e., $P_{5}^{1}$. As illustrated in Figure 5, 5-1, $6-2$ and $7-1$ promptly approach to unity while others diminish to null. From Figure 3, it is apparent that such a routing strategy is indeed a global optimum solution of the routing optimization problem with single-path routing constraints. The learning-based algorithm finds this global optimum solution effectively. Note that this routing strategy is not the only global optimum solution. For instance, $\left(p_{5}^{2}=1, p_{6}^{1}=1\right.$, $p_{7}^{2}=1$ ) is another global optimum solution due to the statistical symmetry of the network. By adjusting the initial conditions, the learning algorithm finds other global optimum routing strategies as well. Duplicate results are omitted.

\section{CONCLUSIONS AND FUTURE WORK}

In this paper, we consider the routing optimization problem in wireless mesh networks under uncertain traffic demands. For multipath routing scenario, we investigate the problem in a stochastic programming framework and a distributed algorithmic solution is derived. For the single-path routing scenario, the problem is formulated as a stochastic integer programming where a learning-based algorithm is proposed.

In our work, we consider a single gateway in the wireless mesh network. The extension to the multiple gateway scenarios remains as future research. In addition, the analysis for the performance of our schemes under non-stationary traffic demand is challenging and needs further investigation.

\section{ACKNOWLEDGEMENT}

This work was supported in part by the U.S. National Science Foundation under Grant DBI-0529012 and under Grant CNS-0721744. 
The work of Fang was also partially supported by Changjiang Scholar Chair Professorship and the 111 Project under B08038.

\section{REFERENCES}

[1] I. F. Akyildiz and X. Wang, "A survey on wireless mesh networks," Communications Magazine, IEEE, vol. 43, no. 9, pp. S23-S30, 2005.

[2] D. Applegate and E. Cohen, "Making intra-domain routing robust to changing and uncertain traffic demands: understanding fundamental tradeoffs," ACM Sigcomm'03, 2003.

[3] D. P. Bertsekas, Nonlinear Programming. Athena Scientific, 1999.

[4] M. Chiang, "To layer or not to layer: Balancing transport and physical layers in wireless multihop networks," IEEE Infocom'04, 2004.

[5] M. Chiang, S. H. Low, A. R. Calderbank, and J. C. Doyle, "Layering as optimization decomposition: A mathematical theory of network architectures," Proceedings of the IEEE, vol. 95, pp. 255-312, Mar.2007.

[6] L. Dai, Y. Xue, B. Chang, and Y. Cui, "Throughput optimization routing under uncertain demand for wireless mesh networks," IEEE Mass'07, 2007.

[7] M. A. Haleem and R. Chandramouli, "Adaptive transmission rate assignment for fading wireless channels with pursuit learning algorithm," Proceedings of CISS, Princeton, 2004.

[8] _ "Adaptive downlink scheduling and rate selection: A cross layer design," IEEE Journal on Selected Areas in Communications, vol. 23, Jun. 2005.

[9] P. Kall and S. W. Wallace, Stochastic Programming. John Wiley \& Sons, 1994.

[10] F. Kelly, A. Maulloo, and D. Tan, "Rate control in communication networks: shadow prices, proportional fairness and stability," Journal of Operation Research Society, vol. 49, pp. 237-252, 1998.

[11] S. Kiran and R. Chandramouli, "An adaptive energy-efficient link layer protocol using stochastic learning control," IEEE International Conference on Communications (ICC), 2003.

[12] L. Kleinrock, Queueing Systems, Volume 1, Theory. Wiley-Interscience; 1 edition, 1975.

[13] J.-W. Lee, R. R. Mazumdar, and N. B. Shroff, “Opportunistic power scheduling for multi-server wireless systems with minimum performance constraints," IEEE Infocom'04, 2004.

[14] _ , "Opportunistic power scheduling for dynamic multi-server wireless systems," IEEE Transactions on Wireless Communications, Jun.2006.

[15] — , "Joint opportunistic power scheduling and end-to-end rate control for wireless ad-hoc networks," IEEE Transactions on Vehicular Technology, Mar. 2007.

[16] Y. Li, B. Bai, J. Harms, and R. Holte, "Multipath oblivious routing for traffic engineering," IEEE/ACM Transactions on Networking, 2007, submitted.

[17] Y. Li, J. Harms, and R. Holte, "Traffic-oblivious energy-aware routing for multihop wireless networks," IEEE Infocom'06, 2006.

[18] A. Medina, N. Taft, K. Salamatian, S. Bhattacharyya, and C. diot, "Traffic matrix estimation: existing techniques and new directions," ACM Sigcomm'02, 2002.

[19] Y. Song, Y. Fang, and Y. Zhang, "Stochastic channel selection in cognitive radio networks," IEEE Globecom'07, 2007.
[20] A. Soule, A. Lakhina, N. Taft, K. Papagiannaki, K. Salamatian, A. Nucci, M. Crovella, and C. Diot, "Traffic matrices: balancing measurements, inference and modeling," ACM Sigmetrics'05, 2005.

[21] M. A. L. Thathachar and P. S. Sastry, Networks of Learning Automata. Kluwer Academic Publishers, 2004.

[22] —_, "Varieties of learning automata: An overview," IEEE Transactions on systems, man, and cybernetics, vol. 32, Dec.2002.

[23] M. H. van der Vlerk, "Stochastic integer programming bibliography," 1996-2007. [Online]. Available: http://mally.eco.rug.nl/biblio/sip.html

[24] H. Wang, H. Xie, L. Qiu, Y. R. Yang, Y. Zhang, and A. Greenberg, "Cope: Traffic engineering in dynamic networks," ACM Sigcomm'06, 2006.

[25] W. Wang, X. Liu, and D. Krishnaswamy, "Robust routing and scheduing in wireless mesh networks," IEEE SECON'07, 2007.

[26] Y. Xing and R. Chandramouli, "Price dynamics in competitive agile spectrum access markets," IEEE Journal on Selected Areas in Communications, Mar.2007.

[27] Y. Xing and R.Chandramouli, "Distributed discrete power control for bursty transmissions over wireless data networks," IEEE International Conference on Communications (ICC), 2004.

[28] G. Zeng, B. Wang, Y. Ding, L. Xiao, and M. Mutka, "Multicast algorithms for multi-channel wireless mesh networks," IEEE ICNP'07, 2007.

[29] J. Zhang, D. Zheng, and M. Chiang, "The impact of stochastic noisy feedback on distributed network utility maximization," IEEE Infocom'07, 2007.

[30] Y. Zhang, M. Roughan, C. Lund, and D. Donoho, "An information-theoretic approach to traffic matrix estimation," ACM Sigcomm'03, 2003. 